\title{
Dynamics of Delayed Neural Networks with Impulses
}

\section{Xiaodi Li*}

School of Mathematical Sciences, Shandong Normal University, Ji'nan, 250014, P.R. China

Neural networks (NNs), especially Hopfield neural networks, Cellular neural networks (CNNs), Cohen-Gross berg neural networks (CGNNs), Bidirectional associative memory (BAM) neural networks, and these networks with time delays, have been deeply investigated in recent years due to their potential applications in the areas of signal and image processing, associative memories and pattern classification, parallel computation and optimization problems. In the design of NNs, the dynamics of networks such as the existence-uniqueness and global asymptotic stability or global exponential stability of equilibrium points of the networks play an important role. For example, in solving optimization problems, the neural network must be designed to have one unique and globally stable equilibrium point. In the analysis of parallel computation, to increase the rate of convergence to the equilibrium point of the networks and reduce the neural computing time, it is necessary to ensure a desired exponential convergence rate of the networks' trajectories, starting from arbitrary initial states to the equilibrium point which corresponds to the optimal solution, and so there is a strong motivation to study the global (exponential) stability of equilibrium points for neural networks $[1,2]$.

Although there are many important results dealing with the static attractors (i.e., equilibrium points) of various NNs, experimental and theoretical studies have revealed that periodic attractors (i.e., stationary oscillation) in NNs is also a very interesting dynamic behavior as many biological and cognitive activities (e.g., heartbeat, respiration, mastication, locomotion, and memorization) exhibit periodicity. Persistent oscillation, such as limit cycles (periodic orbits), represents a common feature of neural firing patterns produced by dynamic interplay between cellular and synaptic mechanisms. Moreover, stationary oscillations in NNs have found many applications such as associative memories, pattern recognition, machine learning, and robot motion control etc. Investigation of stationary oscillations on NNs is indispensable for practical design and engineering applications of network models. Also, an equilibrium point can be viewed as a special periodic attractor of neural networks with arbitrary period. In this sense, the analysis of periodic attractor of neural networks may be considered to be more general than that of equilibrium point. In addition, there are some other dynamics of NNs such as chaos, dissipativity etc. which we shall not discuss here, see the recent book by Zhang [3].

As a kind of dynamic systems, NNs are generally classified into two groups: continuous and discrete networks. Recently, a new system type has been introduced as dynamic systems with impulses, which display some kind of dynamic behavior in a style of both continuous and discrete characteristics, see our recent work by Li [4]. Impulsive phenomenon exists universally in a wide variety of evolutionary processes where the state is changed abruptly at certain moments of time. These include for example many evolutionary processes, particularly some biological systems such as biological neural networks and bursting rhythm models in pathology.

In implementation of electronic networks in which state is subject to instantaneous perturbations and experiences abrupt change at certain moments, which may be caused by switching phenomenon, frequency change or other sudden noise, that exhibit impulsive effects. In fact, when a stimulus from the body or the external environment is received by receptors, the electrical impulses will be conveyed to the neural net and impulsive effects arise naturally. Other examples describing impulsive phenomenon include optimal control models in economics, frequency modulated signal processing systems, and flying object motions. So far the research on dynamics of delayed NNs with impulsive effects has received a great deal of attention and will attract more and more researchers' interest in the future.

\section{References}

1. Arik S (2004) An analysis of exponential stability of delayed neural networks with time varying delays. Neural Networks 17: 1027-1031.

2. Li X, Cao J (2010) Delay-dependent stability of neural networks of neutral-type with time delay in the leakage term. Nonlinearity 23: 1709-1726.

3. Zhang H (2008) Comprehensive analysis and research on dynamical properties of Recurrent delayed Neural Networks. Science Press, Beijing.

4. Li X (2010) New results on global exponential stabilization of impulsive functional differential equations with infinite delays or finite delays. Nonlinear Analysis: Real World applications 11: 4194-4201.
*Corresponding author: Xiaodi Li, School of Mathematical Sciences, Shandong Normal University, Ji'nan, 250014, P.R. China, E-mail: sodymath@163.com

Received June 30, 2012; Accepted July 03, 2012; Published July 06, 2012

Citation: Li X (2012) Dynamics of Delayed Neural Networks with Impulses. J Ap-plied Computat Mathemat 1:e113. doi:10.4172/2168-9679.1000e113

Copyright: ( $2012 \mathrm{LiX}$. This is an open-access article distributed under the terms of the Creative Commons Attribution License, which permits unrestricted use, distribution, and reproduction in any medium, provided the original author and source are credited. 